\title{
A microprocessor-based psychopathology laboratory: IV. Software design
}

\author{
R. S. HUNTZINGER and L. G. SPACE \\ University of Rochester, Rochester, New York 14627
}

\begin{abstract}
Software designed for the microprocessor-based psychopathology laboratory provides a powerful data collection and maintenance package. A library, of prepackaged well-documented program modules has been developed to aid in developing and maintaining programs to administer experiments, to store, retrieve, and interpret data. A simple, but powerful, data base system allows new experiments to be incorporated easily, and obsolete experiments to be deleted. The data collection programs are independent of the general data base, permitting them to be moved to dedicated remote systems as necessary. The data are stored in raw form to permit the researcher to try novel approaches in interpreting existing data.
\end{abstract}

The multifunction psychopathology laboratory is a microprocessor-based testing system capable of administering a variety of experiments. The rationale behind the system, its function, and hardware are described elsewhere (Cromwell \& Spaulding, 1979 ; Space \& Huntzinger, 1979; Spaulding \& Space, 1979). This paper focuses upon the software aspect of the system and the reasoning behind its design. The program development philosophy for this project is discussed first, followed by a description of the programs and data structures involved in the flow of information through the system (Figure 1). The information flow is divided roughly into four stages: (1) collection, (2) storage, (3) retrieval, and (4) interpretation.

\section{PROGRAM DEVELOPMENT PHILOSOPHY}

The psychopathology laboratory will most likely be maintained and operated primarily by persons with limited computer expertise. Due to the rapid turnover of personnel in an academic setting, the authors of programs will often be unavailable for maintenance. For these reasons, it is essential that ease of operation and maintenance be a major consideration in program design.

To meet these needs, the following rules have been adopted: (1) Programs will have a highly modular structure. This permits errors to easily be traced to rather short, somewhat independent blocks of code. (2) Redundant code will be minimized. This shortens programs and avoids the problems of correcting errors replicated throughout the system. (3) Program modules will be self-documenting to aide in identifying the structure and function of the module. (4) Code will be straightforward. Tricky alogrithms are to be avoided. (5) Assembly level code will be minimized. Short as-

Acknowledgment is offered to NIMH Grant MH-14650 and New York State Health Research Council Grant HRC 846. sembly modules are necessary for some "time-critical" applications and to interface peripheral devices to highlevel language programs. More extensive use of assembly language is discouraged. (6) Error diagnostics will be explicit. (7) Programs requesting input from the terminal will output descriptive prompting information. (8) Terminal input will be free format.

The first five programming rules help to provide a library of consistent well-documented programs that are easy to maintain. The last three rules insure that the operator need not act on insufficient information or enter information in an awkward way.

\section{DATA COLLECTION}

The psychopathology laboratory is to collect many different types of data, interacting with an operator and, in most cases, directly with the subject. A series of data collection programs are being developed, each of which collects a specific type of data and stores the results on disk. One collector accepts clinical and demographic

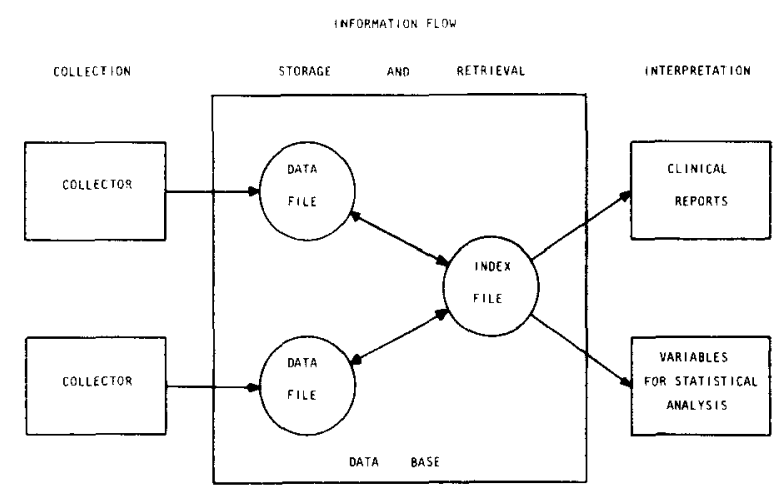

Figure 1. Paths of information flow in the system. Data collectors administer experimental protocols and store data in data files. Using an index file to locate data, the information in the data files is retrieved to prepare clinical reports and variables suitable for statistical analysis. 
data entered by the clerk from the console. Others administer reaction time crossover experiments, span of apprehension, icon decay, the Kelly rep grid, and so on. Each collector is responsible for obtaining one type of data and appending it to the data file associated with that collector.

The data collection programs work independently of each other and the generalized data base. The indepen. dence of the collectors allows us to move the collection programs from our main microcomputer system to remote machines when the work load expands beyond our current limits. Data can be transferred from the remote systems to the central data base on the main system using serial data lines or transfer of disks or magnetic tapes.

Figure 2 shows the structure of a typical data collection program. The entire program administers a complete experimental protocol. The protocol usually consists of two control loops, a practice loop to accustom the subject to the task and an experimental loop in which the data is collected and stored on disk. Both loops have access to common routines that administer an individual trial or request operator intervention. The trial module usually contains the routines that manipulate the experimental apparatus.

The collectors store data in raw form, usually a trialby-trial transcript of the subject's performance. Although this requires more disk space than does the storing of interpreted data, it permits the experimenter to experiment with novel approaches in interpreting previously collected data. In our system, the data are normally interpreted when they are retrieved from the data base.

\section{DATA STORAGE}

The data files maintained by the collector programs have a two-level structure. A low-level block structure is common to all the files. It is used to control file access,

DATA COLLECTION PROGRAM STRUCTURE

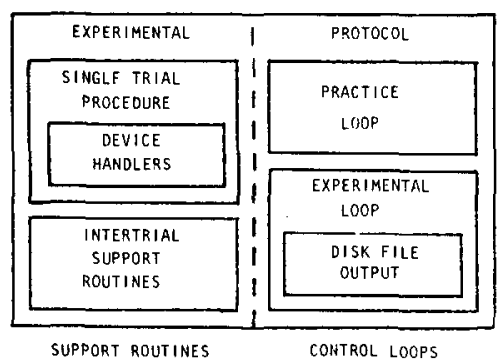

Figure 2. A data collection program administers an entire experimental protocol. This usually involves two control loops, a practice loop to accustom the subject to the task and an experimental loop to collect data. A single trial procedure and various other support routines are available to both control loops.

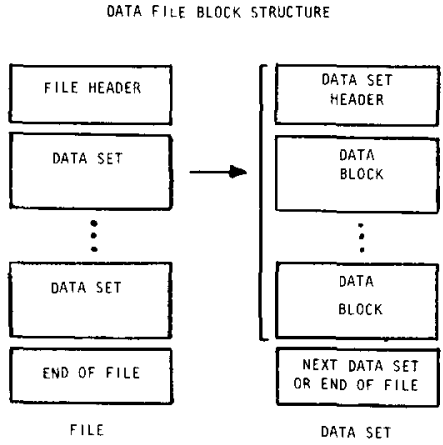

Figure 3. The data files consist of a file header followed by a series of data sets and an end-of-file block. Each data set consists of a header and a series of data blocks.

identify data sets (i.e., records of individual experiments), check errors, and maintain the data base. The block structure permits a common set of program modules to access any data file. The higher level structure is the logical format of the data stored in the file. This structure, unique to each data file, is superimposed on the common block structure.

Figure 3 gives the block structure of our data files. It is similar to the organization used to store data on magnetic tape. The file starts with a header block and is terminated by an end-of-file block. The data sets are stored within this frame, each consisting of a header and a set of data blocks. A data set is terminated by the header of the next data set or the end-of-file block.

Each block in the data file consists of a block length byte, a block type byte, an information field, and a checksum byte. On each access, the program modules responsible for input check the block type (i.e., file header, data set header, data or end-of-file block) and checksum to insure against illegal access. The file header identifies the type of data stored in the file and contains the dates the file was created and last updated and a pointer to the end-of-file block. The pointer is used by the output modules as the address where the next data set is to be written. The end-of-file block stored at that address is always checked before writing to insure that errors in the program or the data file do not cause valuable data to be overwritten.

The data set header identifies the subject, describes the experimental session, and contains a pointer to the next data set (or end-of-file). The subject identification field is given as a "family number" and "individual number" (within family) in anticipation of future genetic studies.

The data blocks contain a variable-length unformatted data field. Information transferred to the file as a continuous stream of binary data is broken into data blocks by the output modules and is unblocked by the input modules upon input. Our FORTRAN data collector programs use a structured data area, such as a common block, to build a high-level data record and 
then call a routine which transfers it to the file as a stream of binary data. To retrieve the structured information, the stream of data is transferred from the file into a common block similar to the one used for writing. Thus, the transfer of data to and from the data files is independent of the format of the data and can be performed by common program modules.

\section{DATA RETRIEVAL}

Retrieval of information from the data base is generally more complex than storage of information in the data base. The data collection programs are only required to append new information to the end of a single data file. On the other hand, programs reading the data base frequently need to access multiple files in random order. For example, we might wish to correlate the reaction time data on a set of subjects with their clinical history. To accomplish this, the reaction time data must be read from one file and the clinical data on the same subject must be read from another file. Because the data files are not ordered, a way of locating the data in each file and accessing it directly is desirable.

To meet these needs, an index file unifies the data base. The index file is simple a directory of the data sets stored in all the data files in the data base. Any number of index files may exist, each capable of indexing up to 254 data files. Each index file and its associated data files make up a data base. If desired, a single data file may be part of more than one data base. Figure 4 shows the structure of an index file. The header identifies the file and contains pointers to the other partitions. The file list contains the name of each data file in the data base and a pointer to the end of the data file when the index was last updated. The rest of the index file consists of index entries, each of which

ACCESSING DATA THROUGH THE INDEX FILE

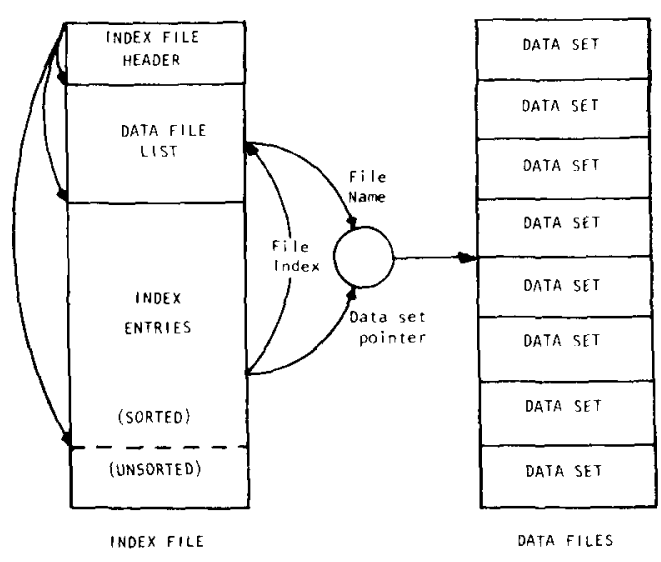

Figure 4. The index file (left) consists of a header, a list of files in the data base, and a series of index entries. To find a data set in the data base (right), file index is used to select the proper file from the file list. A pointer to the data set can then be used to directly access the file. contains the identifier of a specific data set, an index to the file name entry for that data set, a set of flags used for data selection purposes, and a pointer to the data set within the data file. The index entries are stored in two partitions; the first is sorted by data set identifier and data file index, and the other is unsorted. The unsorted partition contains entries added to the file since the last sort.

The index file is built by sequentially reading the data files in the data base and storing the header from each data set and its address in the unsorted index partition. At the end of each file, the end-of-file address is stored in the file name list to use as a starting point for the next update. A sorting program is used periodically to reorder the unsorted partition and merge it with the sorted partition. Because the index is built directly from the data files, new protocols can be added to the data base or old ones excluded by building a new index file.

To access a data set in the data base, the index file is searched for the desired index entry. An efficient binary search is used in the sorted partition and a sequential search in the unsorted partition. When the entry has been found, the data file name index is used to select the proper data file and the pointer to the data set is used to locate the data set within the file (see Figure 4). The program modules that use the index file to access the data base permit up to 15 data sets to be active at a time. The complex data-handling procedures involved in the multifile access system are buried in these modules and transparent to the user.

\section{INTERPRETATION}

In the final stage of information flow through our system, the raw data in the data base are interpreted and the results used to produce data files suitable for statistical analysis or printed reports. The files to be used for statistical analysis can be processed locally or transmitted to a large computer where powerful statistical package programs are available.

Rather than provide a general-purpose interpretative program, we expect the users of the system to write interpretative programs to meet their specific needs. To simplify the preparation of interpretative programs, we provide a hierarchy of prepackaged program modules for accessing the data base and interpretation (Figure 5). When a data collection program is written, an interpretative module is also written which provides a "standard" interpretation of the data stored by that collector. Unless special treatment of the data is required, the user need only provide a control loop that calls each of the desired interpretative modules and the instructions that properly format the output.

\section{SUMMARY}

The software for the microprocessor-based psycho- 
5TRUCTURE OF AN INTERPRETIVE APPLICATIOHS PROGRAM

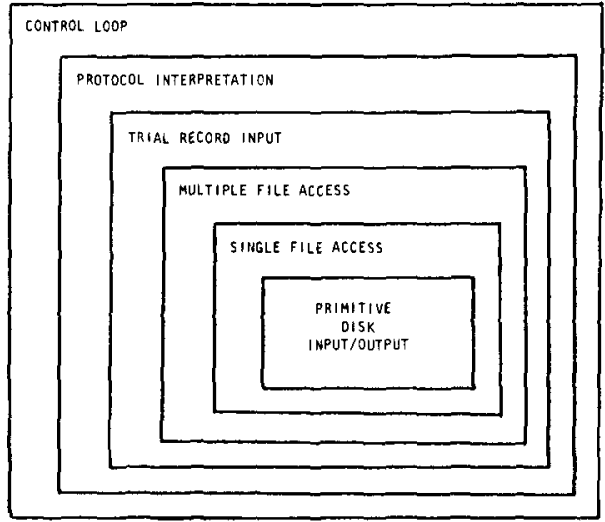

Figure 5. The interpretative programs consist of a hierarchy of program modules used to access data sets in the data base and prepare data for statistical analysis and clinical reports. Prepackaged modules are available for all levels inside the control loop, greatly simplifying program development. pathology laboratory aims to maximize system flexibility while minimizing system complexity and maintenance effort. This has been accomplished with the use of modular programming, independent data collection programs, and a simple indexed data base structure.

\section{REFERENCES}

Cromwell, R. L., \& Spautding. W. D. A microprocessor-based psychopathology laboratory: I. Why bother? Behavior Research Methods \& Instrumentation, 1979, 11, 241-242.

SPACE, L. G., \& Huntzinger, R. S. A microprocessor-based psychopathology laboratory: III. Hardware. Behavior Research Methods \& Instrumentation, 1979, 11, 247-252.

Spaulding, W. D., \& Space, L. G. A microprocessor-based psychopathology laboratory: II. Research goals and design prerequisites. Behavior Research Methods \& Instrumentation, 1979, 11, 243-246. 